\title{
Industrial Application of the Clean Utilization Technology of Arsenic-Alkali Residue with Sb Efficient Enrichment and as Conversion Stabilization
}

ISSN: 2578-0255

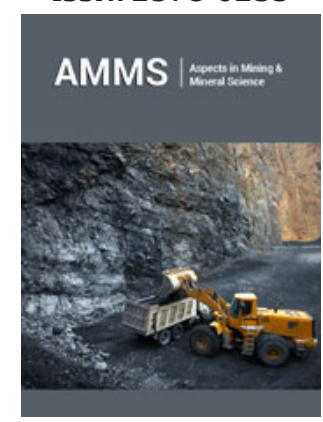

*Corresponding author: Zhiyuan YU, Hunan Provincial Key Laboratory of Water Pollution Control Technology, Hunan Research Academy of Environmental Sciences, Changsha, China

Submission: 阱 November 25, 2019

Published: 眥 December 04, 2019

Volume 4 - Issue 2

How to cite this article: Junqin W, Zhiyuan Y, Erping L, Xishan L, Qingxiong W, et al. Industrial Application of the Clean Utilization Technology of Arsenic-Alkali Residue with Sb Efficient Enrichment and as Conversion Stabilization. Aspects Min Miner Sci.4(2). AMMS.000582.2019.

DOI: 10.31031/AMMS.2019.04.000582

Copyright@ Zhiyuan YU, This article is distributed under the terms of the Creative Commons Attribution 4.0 International License, which permits unrestricted use and redistribution provided that the original author and source are credited.
Junqin $W^{1}$, Zhiyuan $\mathrm{Y}^{2 *}$, Erping $\mathrm{L}^{2}$, Xishan $\mathrm{L}^{3}$, Qingxiong $\mathrm{W}^{3}$ and Shiqiang $\mathrm{T}^{2}$

${ }^{1}$ College of Environmental Science and Engineering, China

${ }^{2}$ Hunan Provincial Key Laboratory of Water Pollution Control Technology, Hunan Research Academy of Environmental Sciences, China

${ }^{3}$ Hunan Chenzhou Mining Limited Liability Company, China

\begin{abstract}
Arsenic-alkali residue is a kind of waste residue produced by removing the arsenic with alkali in the process of refining crude antimony. It is basically stored in the warehouse due to the lack of practical and economic treatment technology. In order to achieve clean utilization and harmless disposal of arsenicalkali residue, a new process of the clean utilization technology of arsenic-alkali residue with Sb efficient enrichment and As conversion stabilization was proposed. Compared with the traditional process, the new one can significantly reduce the As leaching concentration of curing object, and the unit treatment cost will be reduced by $50 \%$. Herein we present a case of industrial application of the clean utilization technology.
\end{abstract}

Keywords: Arsenic-alkaline residue; Clean utilization; Waste treatment by waste; Stabilization treatment

\section{Introduction}

Arsenic-alkali residue is a kind of waste residue produced by removing the arsenic with alkali in the process of refining crude antimony. It contains a lot of highly toxic sodium arsenate and alkali which are easily soluble in water. It will seriously pollute the environment and endanger the health of residents if it is treated improperly [1,2]. Due to the lack of practical and economical treatment technology, arsenic-alkali residue produced by more than $95 \%$ of the enterprises in the smelting industry can only be stored in warehouses, exceeding 500000 tons. The clean utilization and harmless disposal of arsenic-alkali residue is the key factor in determining the sustainable development of the smelting industry [3]. The traditional arsenic-alkali residue treatment process is mainly wet treatment, and the arsenic in the arsenic-alkali residue is produced by sodium arsenate but alkali as sodium carbonate (or sodium hydrogen carbonate), meanwhile the resource utilization rate is low and secondary pollution will be easy to occur. The limited use of sodium arsenate in the industry has hindered the use of arsenic products. At the same time, the sodium carbonate products contain highly toxic sodium arsenate, which is highly soluble in water, resulting in unqualified product quality. The new technology aims at environmental safety and cost reduction from the concept of "Waste Treatment by Waste and Clean Utilization" [4,5]. The key technology research and development of arsenic-alkali residue cleaning and utilization has been carried out based on the research of small test and pilot plant test, and the beneficiation process such as ball milling and re-election is applied to the leaching and enrichment of components in arsenic-alkali residue, attributing to the utilization ratio of arsenic slag is improved [6]. At the same time, using arsenic-containing waste alkali spray to absorb $\mathrm{SO}_{2}$ in the flue gas of smelting blast furnace, to achieve synergistic treatment of arsenic-containing waste alkali and flue gas $\mathrm{SO}_{2}$. Adding sulfuric acid, $\mathrm{H}_{2} \mathrm{O}_{2}$, biological agents and other reagents to the higharsenic wastewater to remove arsenic by oxidation. The slag produced by arsenic removal was treated with Fe-contained stabilizer to meet the requirements of harmless treatment of hazardous waste. In summary, the comprehensive recovery of valuable components in the 
residue like $\mathrm{Sb}$ and alkali and stabilization treatment on As with Fe-contained stabilizer can significantly reduce the treatment cost of arsenic removal slag [7-9], which providing new ideas and new technologies for the resource and harmless treatment of arsenicalkali residue. We report a unique case of industrial application of the clean utilization technology of arsenic-alkali residue with $\mathrm{Sb}$ efficient enrichment and As conversion stabilization.

\section{Case Presentation}

The new technology of the clean utilization technology of arsenic-alkali residue with $\mathrm{Sb}$ efficient enrichment and As conversion stabilization is composed of ball milling leaching, gravity separation and antimony collection, comprehensive utilization of waste alkali, high arsenic wastewater treatment and other systems, whose process flow is shown in Figure 1. The new technology demonstration project had been completed and put into operation in "antimony capital of the world" in January 2019. The treatment scale is $50 \mathrm{t} / \mathrm{d}$, the investment and construction cost is 30 million, and the treatment cost is 2000 yuan/ton, which is $50 \%$ lower than the traditional process treatment cost. The running results showed that the As leaching rate of $96.78 \%$ and alkali leaching rate of $97.35 \%$ could be realized with the liquid-solid ratio of 4:1 grounded in a ball mill and leached for 60 minutes. In order to extract the antimony from the recovered leaching slag, the shaking table was used to recover $\mathrm{Sb}$ with the recovery of $40 \% \sim 50 \%$, and the As content in the concentrate was less than $1 \%$, which could be recycled through the smelting system. Based on the principle of acid-base neutralization, the leachate (high-arsenic waste alkali) entered the flue gas desulfurization spray system in the smelting process, which realized the purpose of synergistic treatment of leaching lye and flue gas $\mathrm{SO}_{2}$.

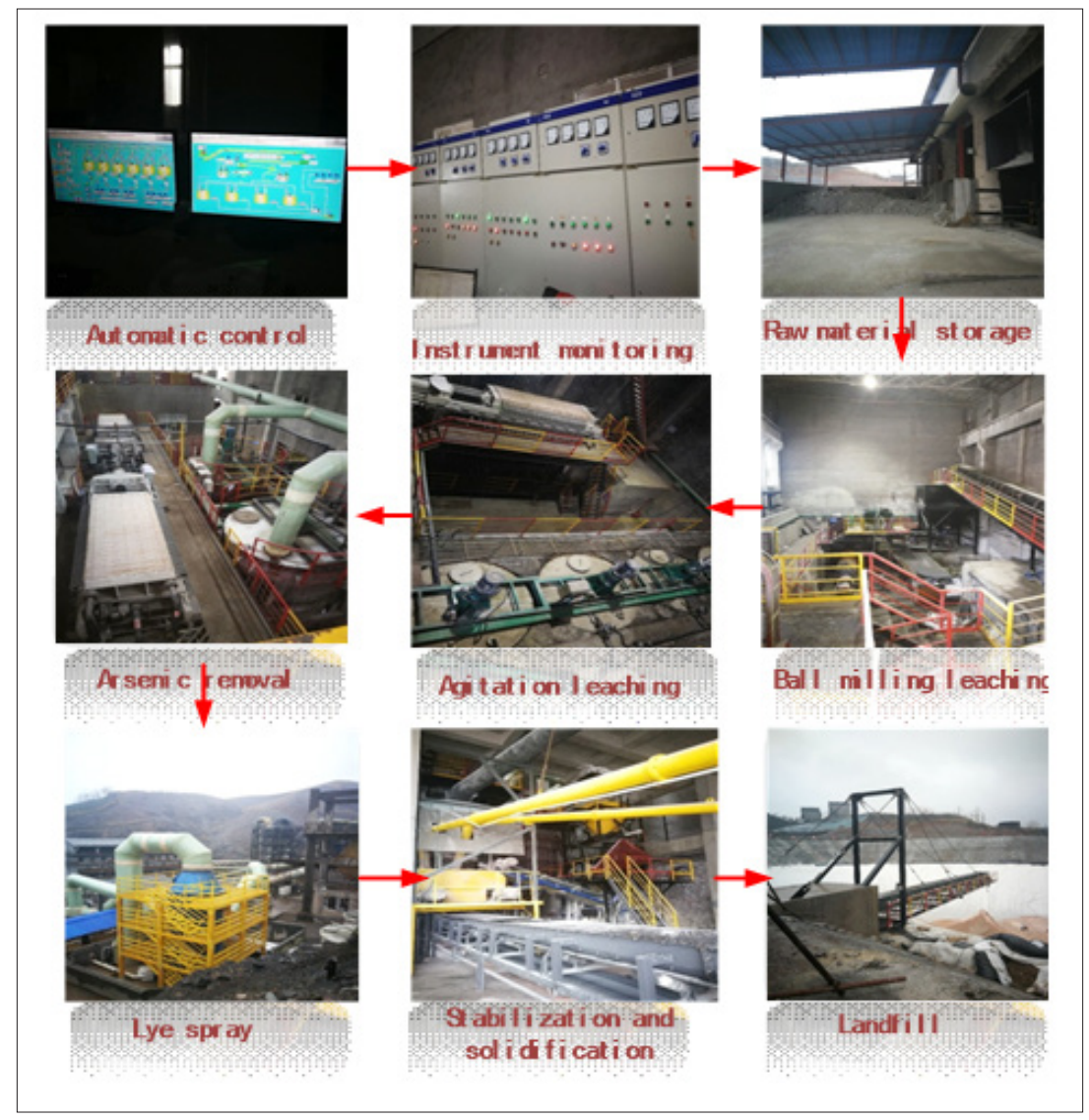

Figure 1: Flow chart of new technology process.

After high-arsenic wastewater was added oxidant $\left(\mathrm{H}_{2} \mathrm{O}_{2}\right)$ and arsenic removal agent (biological agent), the As content in the wastewater was reduced to $150 \mathrm{mg} / \mathrm{m}^{3}$, and the two stages of the arsenic removal efficiency could reach $88.4 \%$ and $92.5 \%$, respectively. The resulting arsenic removal slag was treated with an Fe-contained stabilizer. When the mass ratio of addition was 9\%, the toxicity leaching concentration of the solidified As was reduced from $348.67 \mathrm{mg} / \mathrm{L}$ to $0.65 \mathrm{mg} / \mathrm{L}$, which reached the entry criteria for hazardous waste landfills.

\section{Discussion}

The biggest bottleneck of arsenic-alkali residue treatment is transformation and stable mineralization of harmful element arsenic while ensure harmless disposal of leaching residue when cleaning and recycling valuable element $\mathrm{Sb}$ and waste alkali. First, ensure that all As (III) in the solution is oxidized to As (V), because As (V) is more stable than As (III) and it is uneasy to leach, and then remove As (V) by adding corresponding arsenic removal reagent to make it precipitate with As (V) [10]. The Arsenic removal mechanism was represented in Figure 2. 


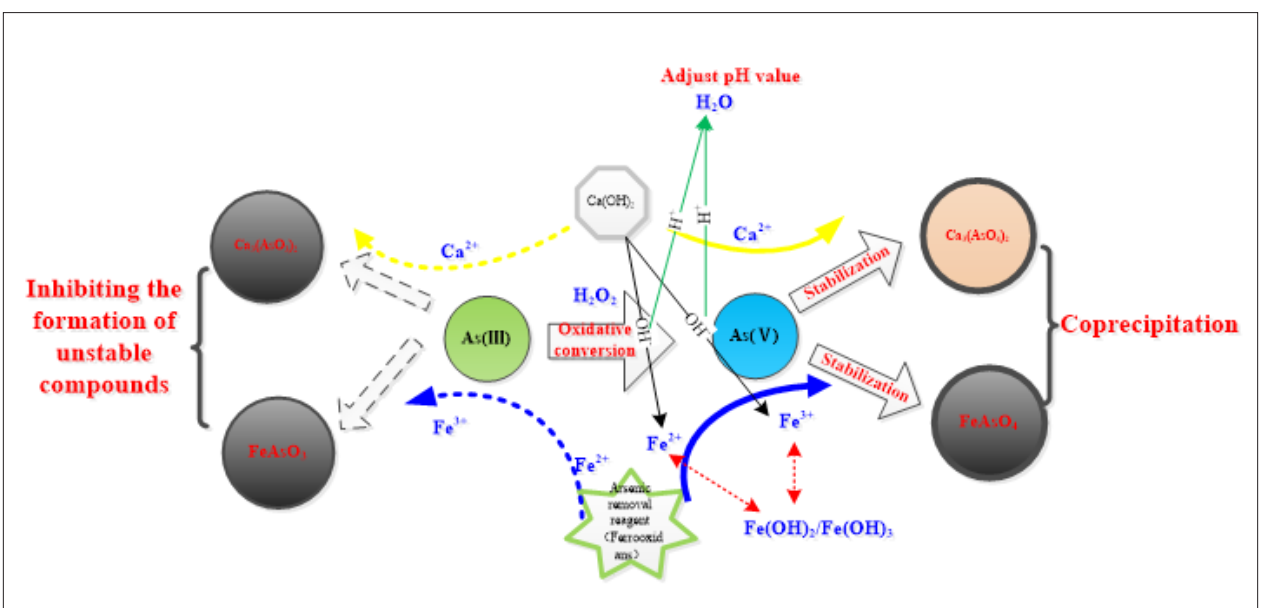

Figure 2: Arsenic removal mechanism.

Then, the arsenic content of the arsenic-alkali residue produced by the precipitation reaction is about $5 \%$, and the leaching concentration of As is more than $2.5 \mathrm{mg} / \mathrm{l}$ under the acid condition so that it cannot be directly landfilled. It needs to be stabilized and pretreated to further stabilize as in the solidified object, so as to prevent the release of As in the long-term stacking process from affecting the ecological environment. According to the principle of geochemistry, the most stable form of As in the earth is scorodite under the condition of weak acid. Therefore, the mixed reaction of stabilizer and arsenic removal residue is used to induce the transformation of As in the arsenic removal residue to scorodite state in the new technology, which can form the most stable state, and significantly reduce the leaching concentration of As. The mineralization mechanism of scorodite, described in Figure 3.

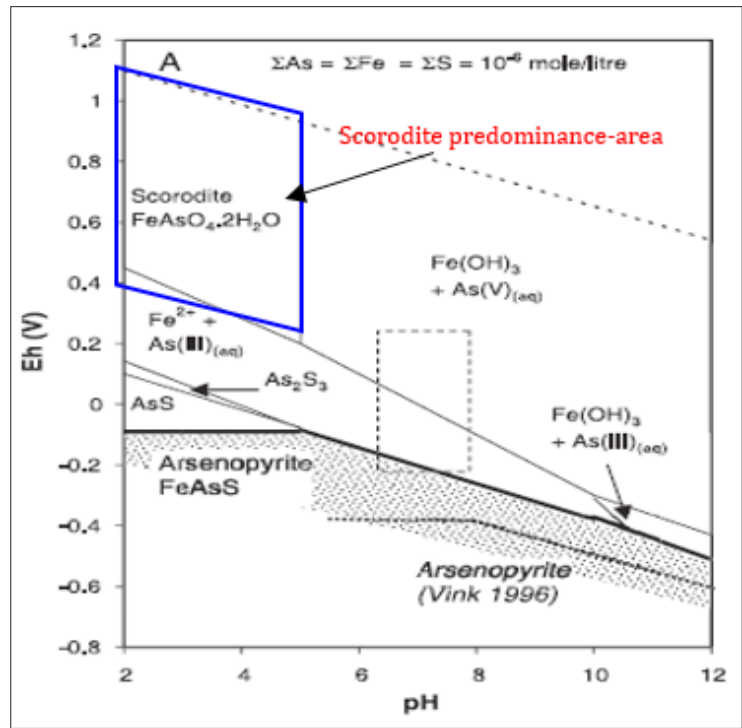

Figure 3: Mineralization mechanism of scorodite predominance-area.

\section{References}

1. Wang Z, Guolong G, Yu M (2013) Harmless disposal and resource utilization technology of arsenic-alkali residue in the smelting industry. Proceedings of the 2013 Annual Conference of the Chinese Society of Environmental Sciences, China.

2. Chen Wei (2016) Separation of arsenic and antimony in arsenic-alkali residue and selective recovery of ruthenium [D]. Kunming: Kunming University of Science and Technology, China.

3. Berdikulova FA, Terlikbayev AZ, Sydykov AO, Mazulevsky EA (2018) Producing metallic antimony with low arsenic content from antimony concentrate. Russian Journal of Non-Ferrous Metals 59(3): 256-260.

4. Deng Weihua (2014) Study on comprehensive recovery of valuable re- sources of arsenic-alkali residue in smelting and smelting [D]. Changsha: Central South University, China.

5. Wenyu W, Wei C, Shunhong H (2015) Pilot study on separation of arsenic and antimony in arsenic-alkali residue. China Nonferrous Metallurgy 44(5): $32-36$

6. Long Hua, Zheng Yajie, Peng Yinglin, Gui-zhong Jin, Wei-hua Deng, et al. (2019) Comparison of arsenic(V) removal with different lead-containing substances and process optimization in aqueous chloride solution. Hydrometallurgy 183: 199-206.

7. Han Feng, Shao Le, Shi Xuefeng (2017) Study on arsenic-alkali residue stabilization curing agent and method. Hunan Nonferrous Metals 33(5): 65-67. 
8. Li Zhiqiang, Chen Wenzhao, Jin Chengyong (2015) Study on the separation of arsenic and alkali by fractional crystallization method. Hunan Nonferrous Metals 31(1): 23-28.

9. Wei Yansong, Yang Zongchao (2014) Experimental study on leaching arsenic from arsenic-alkali residue. Hydrometallurgy 33(5): 371-374.
10. Tong Hui, Liu Chengshuai, Hao Likai (2019) Biological Fe(II) and As(III) oxidation immobilizes arsenic in micro-oxic environments. Geochimica et Cosmochimica Acta 265: 96-108.

For possible submissions Click below: 